\title{
CAVitation Erosion StUdY in DeiOnized WATER Containing Abrasive Particles
}

\author{
Kai Liang Tan, Swee Hock Yeo \\ School of Mechanical and Aerospace Engineering, Nanyang Technological University, 50 Nanyang Avenue, \\ Singapore 639798
}

\begin{abstract}
Cavitation erosion is one of the material removal mechanisms involved in ultrasonic machining (USM). Cavitation bubbles collapse and excite nearby abrasive particles, which at sufficient velocity indent onto workpiece surface, resulting in material loss from the surface. Various studies had been carried out on cavitation erosion in the presence of abrasive particles but only preliminary understanding was achieved. A more thorough understanding about the interaction between bubble collapse and abrasive particles is thus required. In this study, a $20 \mathrm{kHz}$ ultrasonic system was used to generate vibration and cavitation bubbles. Deionized water containing abrasive particles filled the beaker containing the workpiece, positioned at a working gap of $1 \mathrm{~mm}$ from the ultrasonic horn tip. The amplitude of vibration at horn tip was varied from $30-60 \mu \mathrm{m}$ and abrasive concentration varied from $2 \mathrm{wt} \%$ to $10 \mathrm{wt} \%$. SEM images were obtained to observe the surface characteristics and impacts made by abrasive grains under various conditions. Vibration amplitude and abrasive concentration were found to be crucial in affecting extent of cavitation erosion.
\end{abstract}

Keyword: Cavitation erosion; ultrasonic machining; non-contact ultrasonic machining; cavitation damage
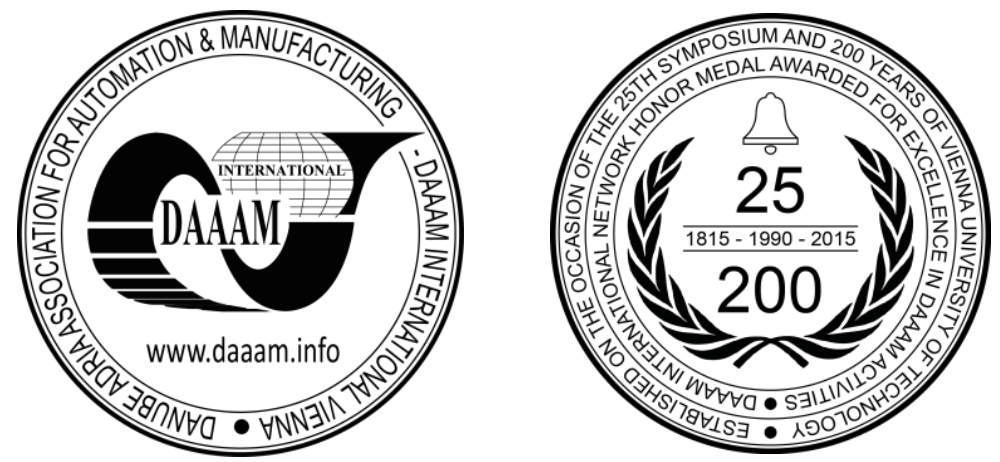

This Publication has to be referred as: Tan, K[ai] L[iang] \& Yeo, S[wee] H[ock] (2016). Cavitation Erosion Study in Deionized Water Containing Abrasive Particles, Proceedings of the 26th DAAAM International Symposium, pp.08180824, B. Katalinic (Ed.), Published by DAAAM International, ISBN 978-3-902734-07-5, ISSN 1726-9679, Vienna, Austria

DOI:10.2507/26th.daaam.proceedings.114 


\section{Introduction}

Cavitation is the formation and activity of bubbles in a liquid. Cavitation bubbles form, grow and collapse due to fluctuations in local liquid pressure. In particular, acoustic cavitation involves sending sound waves in the ultrasonic frequency range $(>20 \mathrm{kHz})$ through a liquid medium. When the negative peak pressure amplitude is brought down to or below the vapor pressure of the liquid, cavities grow and expand [1]. During the cycle of positive pressure amplitude, these bubbles collapse, generating high pressure shockwaves and micro-jets. Erosion, induction of chemical reactions [2] and sonoluminescence [3] are some observed phenomena triggered by bubble collapse.

\subsection{Literature survey}

A solid surface placed near a region of cavitation bubble collapse would undergo microscopic plastic deformation due to high pressure impacts from the micro-jet or micro-shockwave generated [4]. Repeated impacts result in fatigue and subsequent erosion of material from surface. Mechanisms of cavitation erosion and characteristics of cavitation pits produced have been studied extensively [5-8]. Simulation using computational fluid dynamics was also conducted to investigate the speed of micro-jet under various distances of collapsing bubbles from the solid surface [9]. Researchers had also carried out experimental studies on cavitation erosion on some common engineering materials $[10,11]$. However, the behavior of cavitation erosion in the presence of abrasive particles has not been well-understood.

The above-mentioned knowledge could be useful in various fields, such as developing further understanding towards ultrasonic machining (USM). USM is a prominent nontraditional machining technique used to machine hard and brittle materials. It involves introducing ultrasonic vibration to a tool, with abrasive slurry filled in the working gap between the tool and workpiece. Direct hammering of abrasive particles onto the workpiece has been identified as the main mechanism of removal [12]. However, the second mechanism of removal - cavitation erosion, is sometimes overlooked. Despite its small contribution to the total material removal in a USM process, this mode of mechanism could be crucial in the surface integrity and edge quality of the machined products [13]. In addition, improved understanding of this phenomenon is also applicable to the cavitation damage study of hydraulic machinery operating in high-sediment content environment.

It has been reported that in liquid containing particles, the number of cavitation bubbles increases, as the particles' surfaces tend to act as bubble nucleation sites [14]. It is expected that the extent of cavitation erosion is more severe with more bubble collapse. Most studies agree that a larger erosion mass loss could be observed from the presence of abrasive particles [10]. Besides affecting the cavitation bubble population and distribution in a liquid, these particles also take part in material removal directly. Ichida et al. [15] suggested that the collapse of a cavitation bubble excites and accelerates nearby abrasive particles; which in turn impact the workpiece surface and cause abrasive sliding marks. Scanning electron microscopy (SEM) images provided in the study show that the features of abrasive marks are different from erosion pits caused by direct bubble collapse. An experimental study had also been conducted by Huang et al. [16] to study the effect of particle material, size and concentration on cavitation erosion. More recently, Chen et al. [14] investigated the effect of particle shape and concluded that irregular particles tend to result in more abrasive scratches on surface than spherical particles.

Despite the progress, more understanding of the interaction of cavitation bubbles with abrasive particles and characteristics of surface produced could be investigated.

\section{Experimental methodology}

In this study, ultrasonic vibration cavitation erosion experiment was performed in deionized water containing aluminum oxide grits ( 30 $\mu \mathrm{m}$ average size). Based on SEM images and mass loss measurements, the effects of vibration amplitude and abrasive concentration on the extent of cavitation erosion and surface characteristics were investigated.

\subsection{Experimental procedure}

A schematic diagram of the experimental setup is shown in Fig. 1. To produce cavitation bubbles, an ultrasonic generator with $20 \mathrm{kHz}$ frequency (Branson 940) was used. Vibration was transmitted over a transducer and amplified over a booster and horn assembly to have approximately $60 \mu \mathrm{m}$ maximum vibration amplitude at horn tip. The horn tip diameter is $8 \mathrm{~mm}$. Test samples (pure aluminum thin plates) were placed on a holding platform in a beaker and fixed at a distance of $1 \mathrm{~mm}$ from the ultrasonic horn tip. 


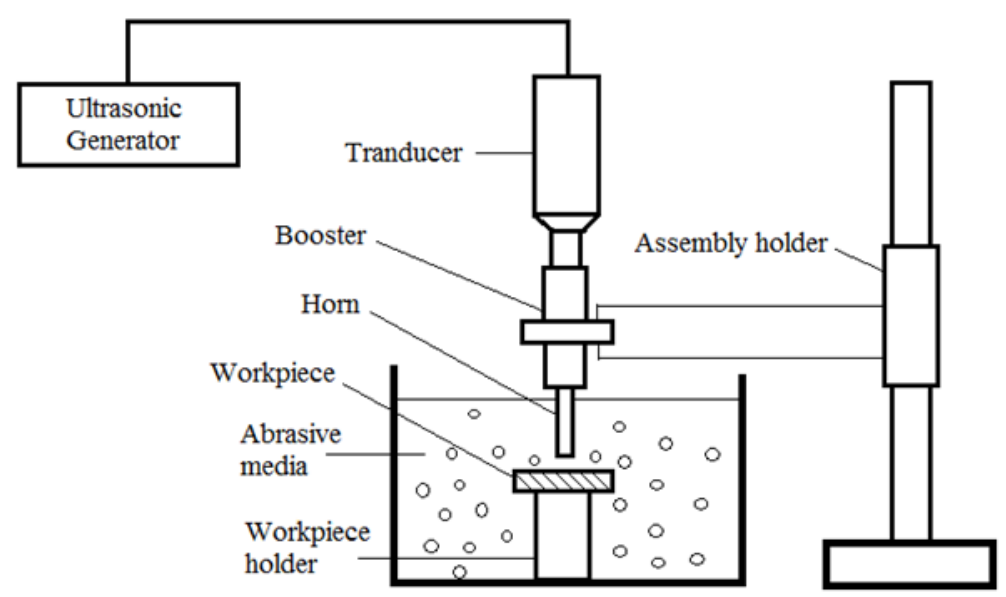

Fig. 1. Schematic diagram of experimental setup

The beaker was then filled with deionized water mixed with hard aluminum oxide abrasive particles (average size $30 \mu \mathrm{m}$ ) of various concentrations at room temperature. Fig. 2 shows the irregular-shaped abrasive particles as observed by SEM (JSM-5600LV, JEOL). During the process, cavitation ensures that the abrasive mixture is heterogeneous, with particles distributed evenly within. Each test sample was subjected to cavitation for 5 minutes; with mass loss being measured after the exposure. SEM images were then obtained to analyze the impacts of abrasive particles on specimens' surface. The experimental conditions are summarized in Table 1.

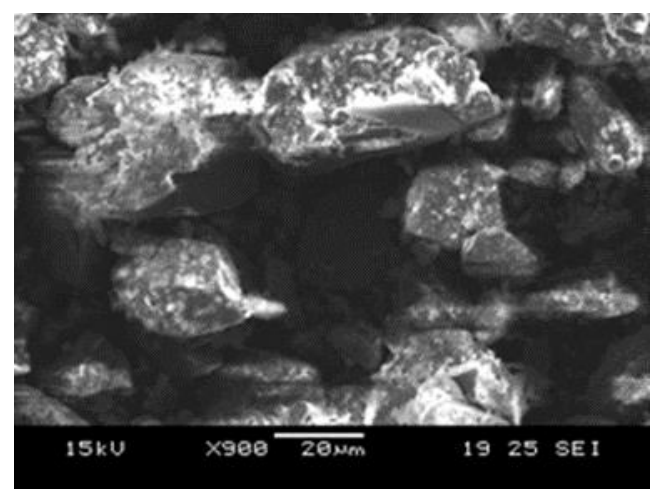

Fig. 2. SEM of aluminum oxide grits used in the experiment

\begin{tabular}{ll}
\hline Experimental conditions & Parameter \\
\hline Ultrasonic frequency $(\mathrm{kHz})$ & 20 \\
Horn tip vibration amplitude $(\mu \mathrm{m})$ & $30 ; 40 ; 50 ; 60$ \\
Liquid media & Deionized water \\
Abrasive particles & $\mathrm{Al}_{2} \mathrm{O}_{3}$ grit \\
Abrasive concentration $(\mathrm{wt} \%)$ & $2 ; 5 ; 10$ \\
Test specimen & $\mathrm{Pure} \mathrm{Al}$ thin plate $(20 \times 20 \mathrm{~mm})$ \\
Clearance $(\mathrm{mm})$ & 1 \\
Machining time $(\mathrm{min})$ & 5 \\
\hline
\end{tabular}

Table 1. Experimental conditions.

\section{Results and discussions}

\subsection{Surface characteristics and mechanism of removal}

Fig. 3a shows the specimen's typical surface characteristics before exposure to cavitation. The specimen surface was polished manually by silicon carbide abrasive papers (grit \#1200), thus a cross-lay pattern could be visible 
across the surface. After exposure to cavitation, the cross-lay pattern disappeared; and the surface was populated by irregularly-sized pits, as shown in Fig. $3 b$.

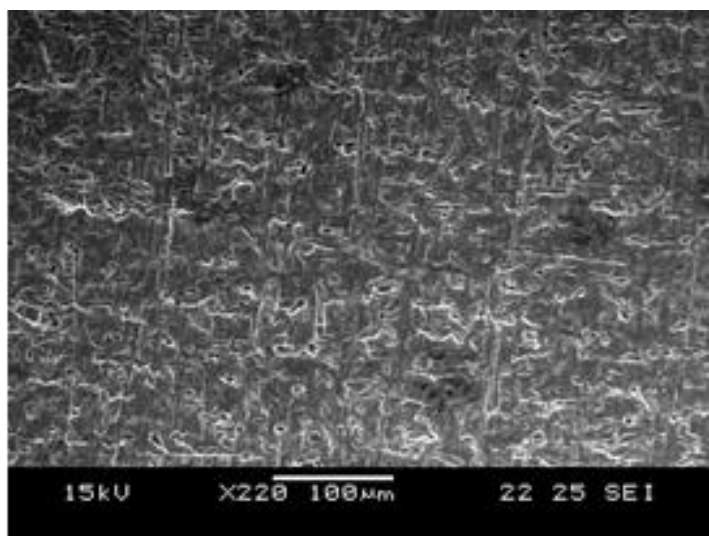

Fig. 3a. Typical cross-lay pattern before cavitation

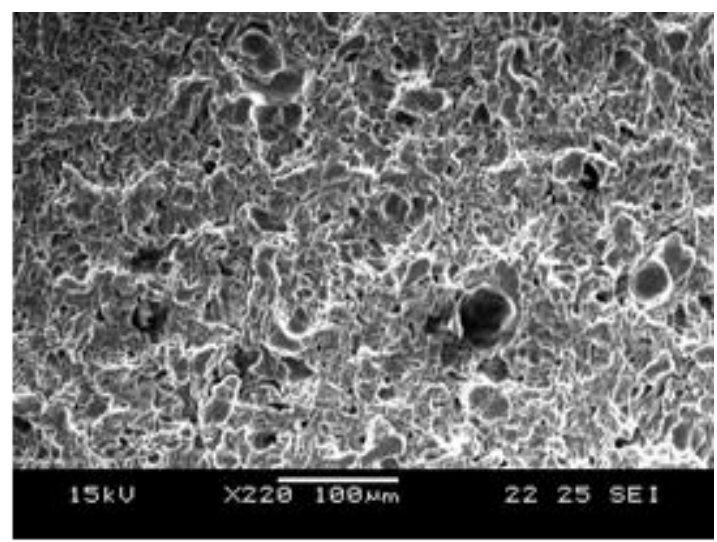

Fig. 3b. Typical eroded surface after $5 \min (5 \mathrm{wt} \%, \mathrm{a}=60$ $\mu \mathrm{m})$

A closer look at the cavitation damages reveals that there are two distinct kinds of erosion pits (Fig. 4). The first type of pits is shallower and generally round-shaped. These damages were mostly caused by direct cavitation bubble collapse near the workpiece surface. The shockwaves emitted from the collapsing bubbles impose high strain on the surface, deforming the surface plastically and creating erosion pits. The second type of damage is represented by deep and irregularly-shaped scratches. These scratches are mostly resulted from the abrasive particles impacting and sliding at high velocity onto the specimen surface.

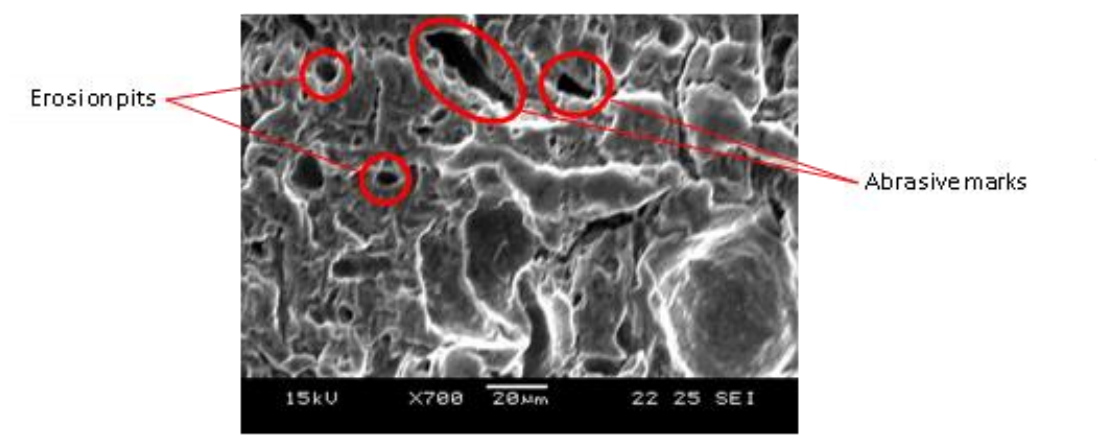

Fig. 4. Erosion pits and abrasive marks observed on specimen surface

Fig. 5 summarizes the two main cavitation erosion mechanisms that contribute to surface modification and mass loss. Bubble collapse near to the specimen surface could cause erosion pits formation; while the micro-shockwave emitted from bubble collapse could also accelerate nearby abrasive particles [17] to impact and abrade specimen surface at high velocity.

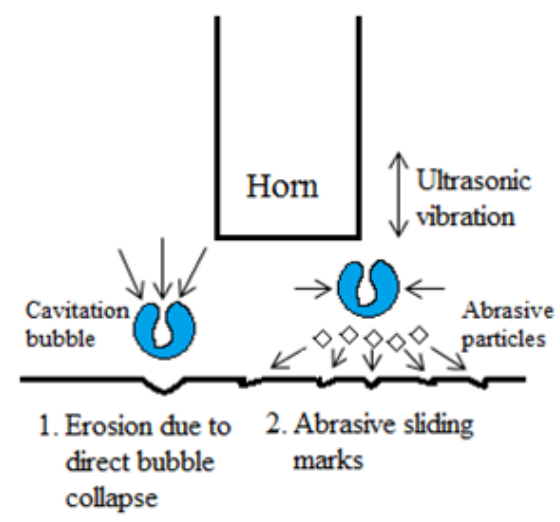

Fig. 5. Illustration of cavitation erosion mechanisms. 


\subsection{Effect of abrasive concentration}

Fig. 6 shows the comparison of workpiece surfaces with different abrasive concentrations used in the experiment. With $2 \mathrm{wt} \%$ abrasive particles, erosion pits are sparse and the original cross-lay pattern is still visible. The number of erosion pits increases drastically when abrasive concentration was altered to $5 \mathrm{wt} \%$ and $10 \mathrm{wt} \%$. This agrees well with existing studies based on mass loss measurements. Increasing abrasive concentration would generally increase the number of cavitation bubbles thus frequency of bubble collapse events. Abrasive particles, particularly irregular particles with rough surfaces, act as nucleation sites for bubble growth.

This hypothesis is further proven by the number of abrasive sliding marks observed. When abrasive concentration was set at $2 \mathrm{wt} \%$, abrasive marks were almost non-existent. On the other hand, extensive abrasive marks were observed on the specimen surfaces with $5 \mathrm{wt} \%$ and $10 \mathrm{wt} \%$ abrasive concentrations. When a further comparison is made between $5 \mathrm{wt} \%$ and $10 \mathrm{wt} \%$ conditions, it could be seen that there is a higher number of embedded abrasive particles observed on the specimen surface of $10 \mathrm{wt} \%$ condition (Fig. 6c). A plausible explanation is that as abrasive concentration increases, the average energy possessed by a single abrasive particle becomes smaller. Hence, abrasives impacted on the surface remain embedded on the surface after the process.

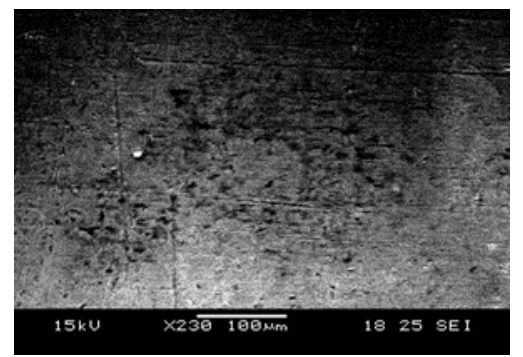

a

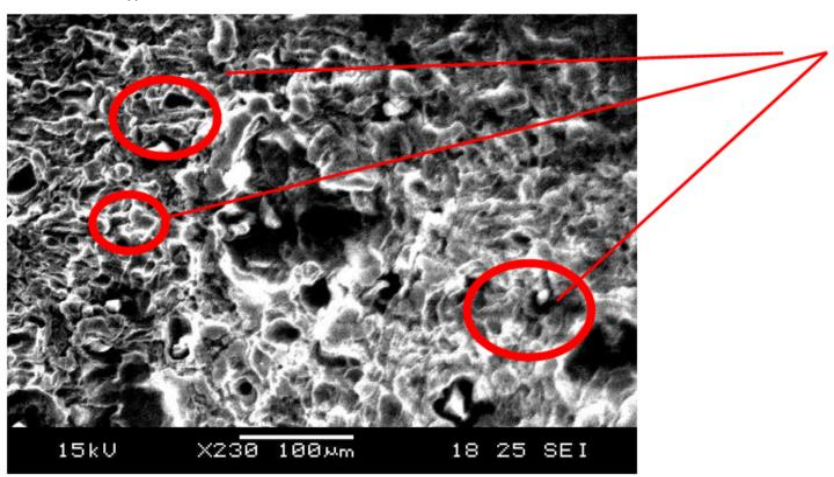

C

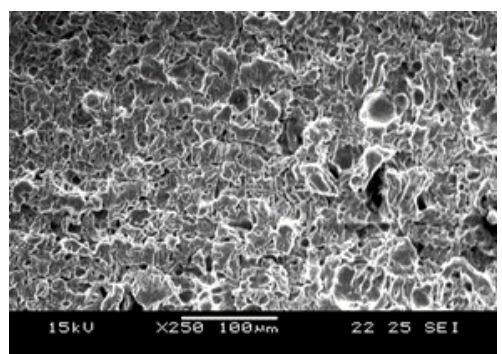

b

Embedded abrasive particles

Fig. 6. Specimen surface after 5 min: a. $2 w t \%, a=50 \mu \mathrm{m} \quad$ b. $5 w t \%, a=50 \mu \mathrm{m} \mathrm{c.} 10 w t \%, a=50 \mu m$

\subsection{Effect of vibration amplitude}

Vibration amplitude of the ultrasonic system is directly related to the number of cavitation bubbles generated and bubble collapse pressure. With smaller amplitudes, the maximum bubble radius becomes smaller, resulting in smaller eventual bubble collapse pressure [18]. As shown in Fig. 7, changes in the surface characteristics were not significant at $30 \mu \mathrm{m}$ vibration amplitudes. Only a few erosion pits were generated; indicating that high energy bubble collapses were rare. Furthermore, a few abrasive particles were found to be embedded onto the specimen surface with no further sliding actions. This could be due to insufficient energy possessed by these particles. With the large abrasive particle size (average diameter $30 \mu \mathrm{m}$ ) used in this experiment, higher vibration amplitude is needed to trigger the abrasives' actions to result in damage on the specimen surface.

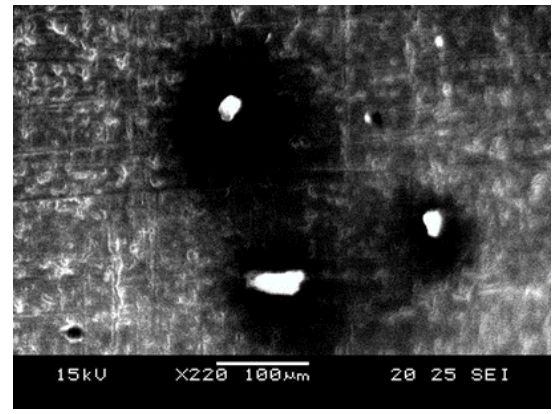

Fig. 7. Specimen surface after $5 \min (5 \mathrm{wt} \%, \mathrm{a}=30 \mu \mathrm{m})$ 


\subsection{Mass loss \& discussion}

Fig. 8 depicts the mass loss of the aluminum specimen at different vibration amplitudes as the abrasive particle concentration in DI water was varied. Mass loss could be attributed to both material removal mechanisms: erosion damage from direct bubble collapse and abrasive indentations. Mass loss increases as the vibration amplitude increases. This further supports the results from SEM images that at higher amplitude of vibration, more cavitation bubbles are nucleated.

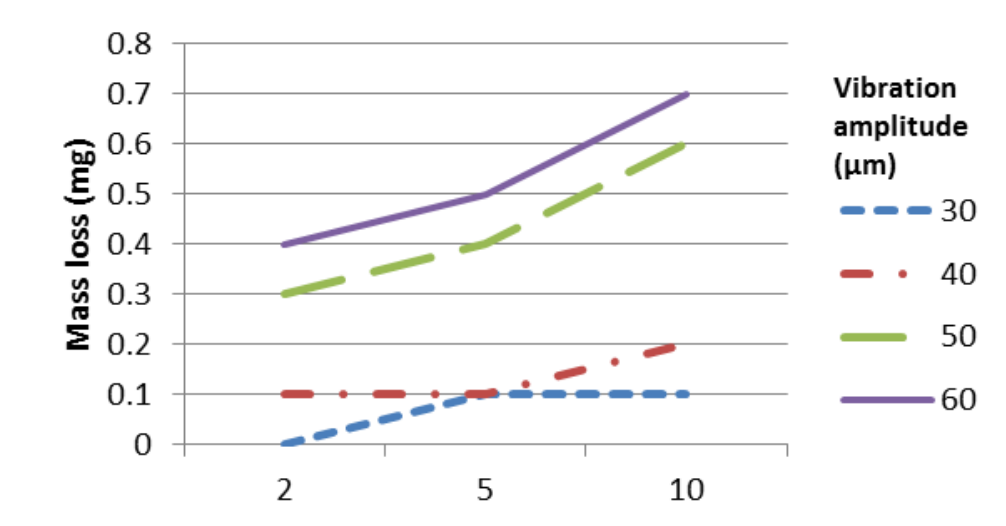

Abrasive concentration (wt\%)

Fig. 8. Graph of specimen's mass loss with varying amplitude and abrasive concentration

However, it could be noted that the mass loss measured is insignificant $(\sim 0.04 \%$ of specimens' mass $)$, thus prone to measurement error. This is due to the short machining time used in this study that corresponds to the incubation stage of cavitation erosion. During the incubation stage, surface material is plastically deformed with insignificant mass loss. Repeated high pressure exerted by the bubble collapse results in work-hardening of the specimen surface and subsequently failure and removal by fatigue [5]. Abrasive sliding marks, on the other hand, also do not contribute much to the mass loss. As the aluminum specimen is ductile, most hard alumina particles impacted and deformed the material surface plastically, creating ridges and crests around the abrasive marks (Fig. 9). Material would only be removed after repeated abrasive impacts, leading to embrittlement of these ridges. These two factors result in mass loss being insignificant and difficult to be detected in this study.

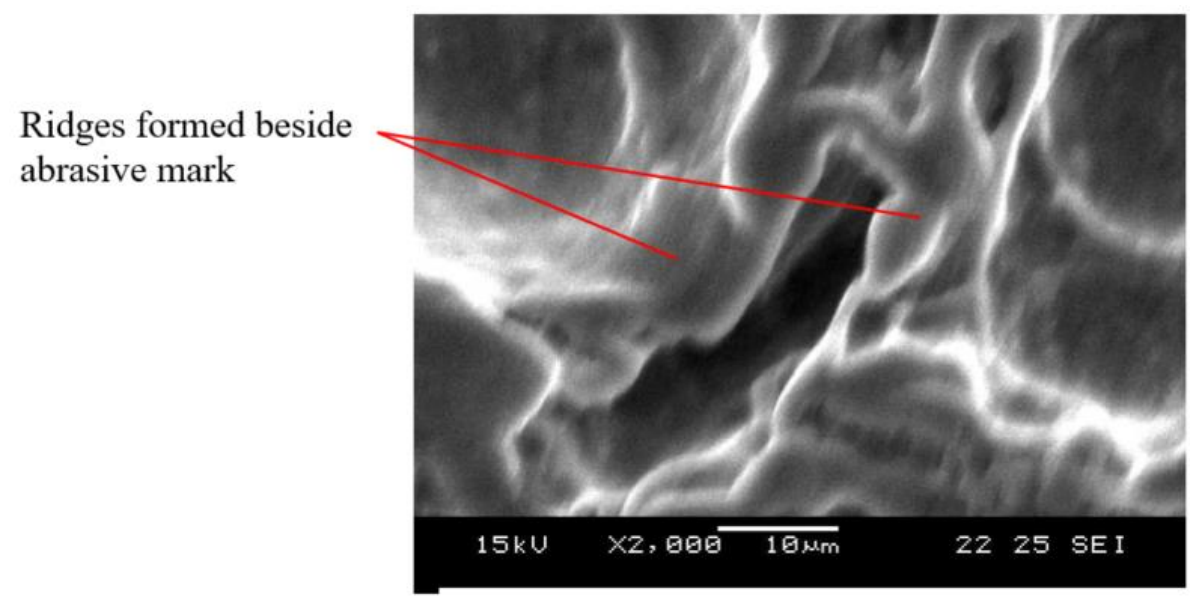

Fig. 9. Magnified view of abrasive sliding mark

\section{Conclusion}

In this paper, cavitation erosion study in deionized water containing hard abrasive particles was conducted. Abrasive particle concentration and vibration amplitude were varied; and the main results are summarized as follows:

- As abrasive particle concentration increases, more cavitation bubbles are nucleated, resulting in more erosion pits. However, the average energy possessed by an individual abrasive particle becomes smaller. 
- With the increase of vibration amplitude, the number of both erosion pits and abrasive sliding marks increases, indicating more cavitation strength.

- Examination of surface characteristics was found to be a good methodology to supplement mass loss evolution in studying cavitation erosion effects.

It has been confirmed that for cavitation in liquid containing hard abrasive particles, two major mechanisms of removal occur. Erosion pits were generated by direct cavitation bubble collapse and abrasive marks were created by abrasive particles impacting the surface at high velocity. Finally, with further explorations in the interaction of cavitation bubbles with abrasive particles, surface integrity and edge quality of USM-ed surface could be better controlled.

\section{Acknowledgements}

This work was conducted within the Rolls-Royce@NTU Corporate Lab with support from the National Research Foundation (NRF) Singapore under the Corp Lab@University Scheme.

\section{References}

[1] F. R. Young, Cavitation, Imperial College Press, London, 1999.

[2] S. J. Doktycz, K. S. Suslick, Interparticle Collisions Driven by Ultrasound. Science (1990) 1067-1069.

[3] W. Lauterborn, T. Kurz, R. Geisler, D. Schanz, O. Lindau, Acoustic cavitation, bubble dynamics and sonoluminescence, Ultrasonics - Sonochemistry 14 (2007) 484-491.

[4] D. G. Shchukin, E. Skorb, V. Belova, H. Mohwald, Ultrasonic Cavitation at Solid Surfaces, Advanced Materials 23 (2011) 1922-1934.

[5] A. Abouel-Kasem, S. M. Ahmed, Cavitation erosion mechanism based on analysis of erosion particles, Journal of Tribology-Transactions of the ASME 130 (2008).

[6] I. Tzanakis, D. G. Eskin, A. Georgoulas, D. K. Fytanidis, Incubation pit analysis and calculation of the hydrodynamic impact pressure from the implosion of an acoustic cavitation bubble, Ultrasonics - Sonochemistry 21 (2014) 866-878.

[7] E. A. Brujan, T. Ikeda, K. Yoshinaka, Y. Matsumoto, The final stage of the collapse of a cloud of bubbles close to a rigid boundary, Ultrasonics - Sonochemistry 18 (2011) 59-64.

[8] M. Dular, O. C. Delgosha, M. Petkovsek, Observations of cavitation erosion pit formation, Ultrasonics Sonochemistry 20 (2013) 1113-1120.

[9] C. Haosheng, L. Jiang, C. Darong, W. Jiadao, Damages on steel surface at the incubation stage of the vibration cavitation erosion in water, Wear 265 (2008) 692-698.

[10] J. R. Laguna-Camacho, R. Lewis, M. Vite-Torres, J. V. Mendez-Mendez, A study of cavitation erosion on engineering materials, Wear 301 (2013) 467-476.

[11] D. Drozdz, R. K. Wunderlich, H. J. Fecht, Cavitation erosion behaviour of Zr-based bulk metallic glasses, Wear 262 (2007) 176-183.

[12] V. Soundararajan, V. Radhakrishnan, An experimental investigation on the basic mechanisms involved in ultrasonic machining, International Journal of Machine Tool Design and Research 26 (1986) 307-321.

[13] C. Nath, G. C. Lim, H. Y. Zheng, Influence of the material removal mechanisms on hole integrity in ultrasonic machining of structural ceramics, Ultrasonics 52 (2012) 605-613.

[14] C. Haosheng, W. Jiadao, C. Darong, Cavitation damages on solid surfaces in suspensions containing spherical and irregular microparticles, Wear 266 (2009) 345-348.

[15] Y. Ichida, R. Sato, Y. Morimoto, K. Kobayashi, Material removal mechanisms in non-contact ultrasonic abrasive machining, Wear 258 (2005) 107-114.

[16] S. Huang, A. Ihara, H. Watanabe, H. Hashimoto, Effects of solid particle properties on cavitation erosion in solidwater mixtures, Journal of Fluids Engineering 118 (1996) 749-755.

[17] O. V. Abramov, High-intensity ultrasonics : theory and industrial applications, Gordon and Breach Science Publishers, Amsterdam, The Netherlands, 1998.

[18] T. G. Leighton, The acoustic bubble: Academic Press, London, 1994. 